\title{
Effective interactions in the stripe state of the two-dimensional Hubbard model
}

\author{
S. Varlamov and G. Seibold \\ Institut für Physik, BTU Cottbus, PBox 101344, 03013 Cottbus, Germany
}

(October 22, 2018)

\begin{abstract}
We investigate the structure of the pairing potential in the stripe phase of the two-dimensional Hubbard model. Based on the random phase approximation we discuss in detail the interactions in the charge- and spin channel and compare our calculations with related considerations in commensurate antiferromagnets. Our main finding is that due to the incommensurate charge-density wave formation the exchange of collective modes in the charge channel is significantly enhanced with respect to the spin bag approach whereas due to the inhomogeneous charge distribution the coupling to transverse spin fluctuations tends to be suppressed.
\end{abstract}

\section{INTRODUCTION}

The existence of electronic inhomogeneities is now a well established experimental fact in underdoped high $\mathrm{T}_{c}$ materials (see e.g16). Especially in lanthanum cuprates these inhomogeneities can develop in the form of antiphase domain walls where quasi one-dimensional charge stripes are separated by hole-free antiferromagnetically $(\mathrm{AF})$ ordered regions. It is worth to note that stripe textures have been predicted as stable HartreeFock (HF) saddle-points of the Hubbard model 8 before they were found experimentally in cuprates and nickelates. Stripe textures in high- $\mathrm{T}_{c}$ materials have been first detected in Nd-doped lanthanum cuprate by neutron scattering experiments where due to the occurence of a low temperature tetragonal phase (LTT) both incommensurate AF and charge order are pinned 9 . The relevance of structural distortions for the formation of antiphase domain walls has been recently studied in Ref.10. In Nd-free compounds 11,12 these measurements have up to now only detected the magnetic part of the scattering which remarkably displays the same incommensurability than in the Nd-doped system. In addition evidence for incommensurate charge fluctuations comes from the analysis of optical phonon measurements in $\mathrm{YBa}_{2} \mathrm{Cu}_{3} \mathrm{O}_{6.6} \mathrm{H3}$ and neutron diffraction experiments 14 in $\mathrm{La}_{2-x} \mathrm{Sr}_{x} \mathrm{CuO}_{4}$ (LSCO). Taking the stripes as an experimental fact the question arises wether they have something to do with high- $\mathrm{T}_{c}$ superconductivity or if they are just some 'strange byproduct' of the strong correlations which are at work in these materials. In fact there are many experiments which support the point of view that stripe correlations could give rise to high transition temperatures. First, it has been shown that the stripe spacing in LSCO compounds is inversely proportional to the transition temperature up to optimal doping 11 . A close connection between transition temperature and stripe incommensurability has also been found in YBCO15 where interestingly there seems to appear a discontinuous jump of incommensurability from $1 / 6$ at optimal doping to $1 / 8$ for the $\mathrm{T}_{c}=60 \mathrm{~K}$ underdoped compound. Second, the occurence of domain walls can be imterpreted in terms of a quantum critical point (QCP) 16 located near opti- mal doping $\mathrm{x}_{\text {opt }}$ which is a quite appealing concept in order to account for the anomalous normal state transport of high- $\mathrm{T}_{c}$ materials around this particular doping. As a consequence the QCP scenario allows for a natural subdivision of the phase diagram into overdoped metallic and the underdoped pseudogap phase. It has been argued 17 that the QCP can be deduced from the amplitude of the incommensurate magnetic peaks vansishing near $\mathrm{x}_{\text {opt }}$. This coincides with experiments where superconducting order has been suppressed by strong pulsed magnetic fields 18 and which have revealed an underlying metal insulator transition at about the same concentration in agreement with the QCP concept. Temperature dependent measurements of the charge and spin sector in Nd-doped LSCO' as well as NQR experiments 6 further support the idea that it is the charge rather than the spins which is reponsible for the stripe state instability.

The possibility of d-wave pairing near a incommen surate CDW instability has been investigated in Ref. 19 within a BCS-type scheme. This analysis was restricted to the overdoped and optimally doped region of the phase diagram where according to the QCP scenario strong ICDW fluctuations are present but without symmetry breaking of the translational invariance. In the underdoped system the opening of a ICDW gap naturally leads to a suppression of the superconducting order parameter due to a reduction in density of states around the Fermi level (see e.g.20,21). However, in addition the ICDW fluctuations are strongly modified in the symmetry-broken state but to our knowledge no detailed analysis of the corresponding pairing interaction has been performed yet. The present paper is dedicated to explore the effective electron-electron interactions in the underdoped regime of the phase diagram thus starting with a ground state which exhibits long-range incommensurate stripe order. Our considerations are based on the Hartree-Fock (HF) decoupled two-dimensional Hubbard model where we obtain the domain wall structure via a self-consistent iteration of the on-site charge and spin expectation values. From the formal point of view our investigations can be viewed as a generalization of the spin-bag approach 22 to incommensurate antiferromagnetism. The basic idea of the spin-bag mechansim is that a hole doped into the commensurate antiferromag- 
net locally perturbes the AF order parameter and consequently two holes gain energy when they share a common deformation (hag) in analogy to a standard lattice bipolaron. In Ref 22 this scenario has been worked out by considering the pairing in the longitudinal spin channel of a commensurate antiferromagnet which indeed turns out to be attractive for small momentum transfers whereas it becomes repulsive for large $\mathbf{q} \sim(\pi, \pi)$. The charge channel plays a minor role for pairing in the spin-bag approach since it does not exhibit an instability and renormalization effects are rather weak. It has been argued in Ref.22 that the repulsion in the transverse spin channel at large q may be suppressed by the coherence factors entering the vertex of the pairing interaction. However, as pointed out by Frenkel and Hanke 23 the vanishing of the vertex is cancelled by the Goldstone pole in the spin susceptibility thus leading to a finite repulsive contribution in the transverse channel for momenta $\mathbf{q} \sim(\pi, \pi)$ comparable to that for the amplitude fluctuations.

We want to emphasize that our HF approach to the stripe phase fails to account for some of the experimental facts mentioned above but on the other hand allows for a simple description of some basic features regarding the electron-electron interaction in a sutem posessing stripe order. First, it is well known 24 that a $\mathrm{HF}$ decoupling of the one-band Hubbard model cannot properly describe the observed stripe charge structure in the cuprates which is characterized by one hole for every second unit cell along the domain wall ('half-filled'). In contrast $\mathrm{HF}$ calculations result in completely filled stripes thus predicting twice the observed stripe periodicity and it has turned out that correlations beyond HF theory have to be included 25,26 in order to account for the measured stripe structure within the Hubbard model.

Thus in our investigations we will restrict to excitations perpendicular to the domain walls which should be less affected by the actual stripe filling than excitations along the walls which of course crucially depend on the doping of the stripes. Second, it has been shown in Ref.27 that the HF stripe instability is due to an instability in the spin channel and the corresponding charge modulation is generated as a higher harmonic of the longitudinal spin scattering. Thus in our model the spins are the driving force behind the domain wall formation in contrast to what is observed in Nd-doped LSCO. An alternative approach where antiphase domain walls are enslaved by a frustrated phase seperation scenario has been discussed in 29. However, since we restrict on zero temperature we believe that within the limitations discussed above our approach can capture some features of the electron-electron interaction in the stripe state of underdoped cuprates. We note that a similar study for the calculation of correlation functions has been performed in Ref.28 where the authors focussed on the renormalization of the spin velocity in the stripe state.

The paper is organized as follows. In Sec. II we outline the formalism of our approach starting from HF theory and the subsequent derivation of effective hamiltoni- ans for the residual RPA-type interactions in the stripe phase. In Sec. III we present results for these interactions focussing on the different contributions from vertex functions and susceptibilities respectively. We finally conclude our discussion in Sec. IV.

\section{FORMALISM}

\section{A. Mean-field theory}

We start with the Hubbard hamiltonian

$$
H=\sum_{k, \sigma} \epsilon_{k} c_{k, \sigma}^{\dagger} c_{k, \sigma}+\frac{U}{2} \sum_{q, \sigma} \rho_{-q,-\sigma} \rho_{q, \sigma}
$$

where $c_{k, \sigma}^{(\dagger)}$ destroys (creates) an electron in the state $\mathrm{k}$ and energy $\epsilon_{k}, \rho_{q, \sigma}=\sum_{k} c_{k+q, \sigma}^{\dagger} c_{k, \sigma}$ denotes the density operator.

For one elecron per site the ground state shows twosublattice commensurafe antiferromagnetic order. It has been shown by Schul2 27 using the RPA-type Stoner criterion that for a small number of holes the system posesses a magnetic instability at an incommensurate modulation wavevector $(\pi, \pi)+\mathbf{q}_{\mathbf{s}}$. In principle the corresponding (mean-field) symmetry-broken state could be realized either by a modulation of the transverse spin degrees of freedom i.e. a spiral phase 30 or the corresponding modulation of the longitudinal magnetization. From general argument\$31 and also from unrestricted Hartree-Fock calculations (see e.g.24) one finds that usually the symmetry breaking occurs in the longitudinal channel of the magnetic system. As a consequence this leads to a strong coupling between charge- and spin degrees of freedom resulting in the formation of antiphase domain walls where the charge is distributed along the phase boundaries. It should be noted that the inclusion of the vacuum renormalization of the effective interaction may stabilize the commensurate antiferromagnet at small dopings 32 .

Within unrestricted HF theory (see e.g. 33 ) one finds that for small values of the on-site interaction $U$ the stripes are oriented along the verticals and whereas for large $\mathrm{U}$ they run along the (1,1)-direction. In the following we consider a rather small value of $U$ close to the instability in order to realize the vertical stripe phase.

Moreover we restrict ourselves to an energy dispersion arising from nearest neighbor hopping $\varepsilon_{k}=-2 t\left(\cos k_{x}+\right.$ $\cos k_{y}$ ) with bandwidth $B=8 t=4 e V$. For doping $\delta=0.13$ (measured from half-filling) one finds from the Stoner criterion the occurence of the magnetic instability at $\mathbf{Q}^{\mathbf{t}}=\pi(1 \pm 1 / 8,1)$ and $\mathbf{Q}^{\mathbf{t}}=\pi(1,1 \pm 1 / 8)$ when the Hubbard interaction exceeds the critical value $\mathrm{U}_{c} \sim 0.9 \mathrm{eV}$. In principle these four equivalent wave vectors allow either for an unidirectional stripe structure or for a two-dimensional grid pattern of antiphase domain walls. In the spin glass phase of LSCO where the stripes 
run along the diagonals neutron scattering provides convincing evidence for a one-dimensional modulation 37 , however, in the superconducting regime this pgint remains unclear although from general arguments38 unidirectional scattering seems to be favored.

Restricting ourselves to the (longitudinal) domain wall structure the Hubbard interaction can be decoupled via

$$
\begin{aligned}
\rho_{-q,-\sigma} \rho_{q, \sigma} & \approx \frac{1}{4}\left(\left\langle\rho_{-q}\right\rangle \rho_{q}+\left\langle\rho_{q}\right\rangle \rho_{-q}-\left\langle\mu_{-q}\right\rangle \mu_{q}-\left\langle\mu_{q}\right\rangle \mu_{-q}\right) \\
& -\frac{1}{4}\left(\left\langle\rho_{q}\right\rangle\left\langle\rho_{-q}\right\rangle-\left\langle\mu_{q}\right\rangle\left\langle\mu_{-q}\right\rangle\right)
\end{aligned}
$$

where $\left\langle\rho_{q}\right\rangle=\sum_{\sigma}\left\langle\rho_{q, \sigma}\right\rangle$ and $\left\langle\mu_{q}\right\rangle=\sum_{\sigma} \sigma\left\langle\rho_{q, \sigma}\right\rangle$ denote the charge and spin densities respectively. Although the Stoner criterion signals the instability at a single wave vector $\mathbf{Q}^{\mathbf{t}}=(\pi, \pi)+\mathbf{q}_{\mathbf{s}}$ the anharmonicity of the domain wall structure in the symmetry broken phase naturally incorporates higher harmonics also. It is well known 34 that the topological nature of the stripe phase implies the relation $\mathbf{q}_{\mathbf{c}}=2 \mathbf{q}_{\mathbf{s}}$ between the basic charge $\left(\mathbf{q}_{\mathbf{c}}\right)$ and $\operatorname{spin}\left(\mathbf{q}_{\mathbf{s}}\right)$ modulations.

In order to illustrate the role of these harmonics in the charge- and spin channel respectively, we have sketched in Fig. 1 the charge $(\chi(x))$ and spin order $(\Delta(x))$ parameter for a simple rectangular stripe pattern in the periodicity interval $[0, l]$. One can easily convince oneself that the corresponding Fourier coefficients $\chi_{k}$ and $\Delta_{k}$ are given by

$$
\begin{aligned}
\chi_{k} & =\frac{4 \chi_{0}}{k \pi} \cos (k \pi / 2) \cos \left(\frac{k \pi \xi}{l}\right) \\
\Delta_{k} & =\frac{4 \Delta_{0}}{k \pi} \sin (k \pi / 2) \sin \left(\frac{k \pi \xi}{l}\right)
\end{aligned}
$$

where $\xi$ denotes the width of the magnetic domains. Thus the magnetic scattering only involves odd harmonics whereas the charge scattering is due to the even harmonics. This fact holds in general when both magnetic and charge stripes are symmetric under reflection with respect to their central axis. As a result one can describe the modulation of charge and spin densities in terms of the single wave vector $\mathbf{Q}^{\mathbf{t}}$

$$
\begin{aligned}
& \langle\rho(\mathbf{r})\rangle=2 \sum_{p(\text { even })=2}^{M-1}\left\langle\rho_{p} \mathbf{Q}^{\mathbf{t}}\right\rangle \cos \left(p \mathbf{Q}^{\mathbf{t}} \mathbf{r}\right), \\
& \langle\mu(\mathbf{r})\rangle=2 \sum_{p(\text { odd })=1}^{M-1}\left\langle\mu_{p} \mathbf{Q}^{\mathbf{t}}\right\rangle \sin \left(p \mathbf{Q}^{\mathbf{t}} \mathbf{r}\right)
\end{aligned}
$$

where the commensurability $M$ of the stripe is defined as the number of scattering events in the same direction needed to return to the equivalent state, i.e. $\left|\mathbf{k}+M \mathbf{Q}^{\mathbf{t}}\right\rangle=|\mathbf{k}\rangle$. Note that within this definition a large commensurability $\mathrm{M}$ not necessarily implies a scattering vector $\mathbf{Q}^{\mathbf{t}}$ very distant from $(\pi, \pi)$.

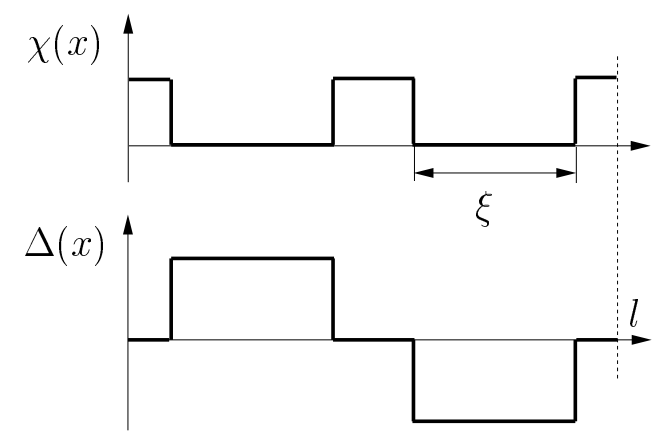

FIG.1. Illustrative plot for the charge $(\chi(x))$ and spin order $(\Delta(x))$ parameter of a rectangular stripe pattern.

For the vertical stripe orientations along the $\mathrm{x}$ direction considered here we can write $\mathbf{Q}^{\mathbf{t}}=\pi\left(\frac{2}{M}+\right.$ $\Theta(M-2), 1)$ where the step function is defined with $\Theta(0)=0$. This guarantees the correct behavior for commensurate $\operatorname{AF}(\mathrm{M}=2)$ i.e. $\mathbf{Q}^{\mathbf{t}}=(\pi, \pi)$. Note that within these definitions the commensurability $\mathrm{M}$ corresponds to the period of the stripe structure (in units of the lattice constant) as is indicated in Fig. 2a. Furthermore we are now able to construct the reduced Brillouin zone (BZ) which is spanned by vectors $\mathbf{Q}^{\mathbf{t}}$ and $\mathbf{Q}_{\perp}=\frac{4 \pi}{(2+M \Theta(M-2))^{2}+M^{2}}(-M, 2+M \Theta(M-2))$. In order to illustrate the new zone scheme we plot in Fig. $2 \mathrm{~b}$ the reduced $\mathrm{BZ}$ for the case $M=16$ which will be studied below. Note that the reduced BZ is not only due to charge order (the corresponding vertically striped BZ is also indicated in Fig. 2b) but is rotated and divided in half due to the presence of (modulated) antiferromagnetism.

Introducing reduced zone notation for the electron operators

$$
c_{n}(k, \sigma) \equiv c_{k_{x}+(n-1) Q_{x}^{t}, k_{y}+(n-1) Q_{y}^{t}, \sigma}
$$

we thus obtain the following hamiltonian

$$
\begin{aligned}
H & =\sum_{n, k, \sigma} \epsilon_{n}(k) c_{n}^{\dagger}(k, \sigma) c_{n}(k, \sigma) \\
& +\sum_{p, n, k, \sigma}\left[\chi_{2 p} c_{n+2 p}^{\dagger}(k, \sigma) c_{n}(k, \sigma)+h . c .\right] \\
& +\sum_{p, n, k, \sigma} \sigma\left[\Delta_{p} c_{n+p}^{\dagger}(k, \sigma) c_{n}(k, \sigma)+h . c .\right] \\
& +\frac{2 N}{U} \sum_{p}\left(\left|\Delta_{p}\right|^{2}-\left|\chi_{2 p}\right|^{2}\right)
\end{aligned}
$$

where we have introduced the charge and spin order parameters as $\chi_{2 p}=(U / 2)\left\langle\rho_{2 p} \mathbf{Q}^{\mathbf{t}}\right\rangle$ and $\Delta_{p}=$ $-(U / 2)\left\langle\mu_{p} \mathbf{Q}^{\mathbf{t}}\right\rangle$.

The hamiltonian eq. (6) can be diagonalized via

$$
c_{n}(k, \sigma)=\sum_{m=1}^{M} A_{n m}(k, \sigma) f_{m}(k, \sigma)
$$


$(a)$
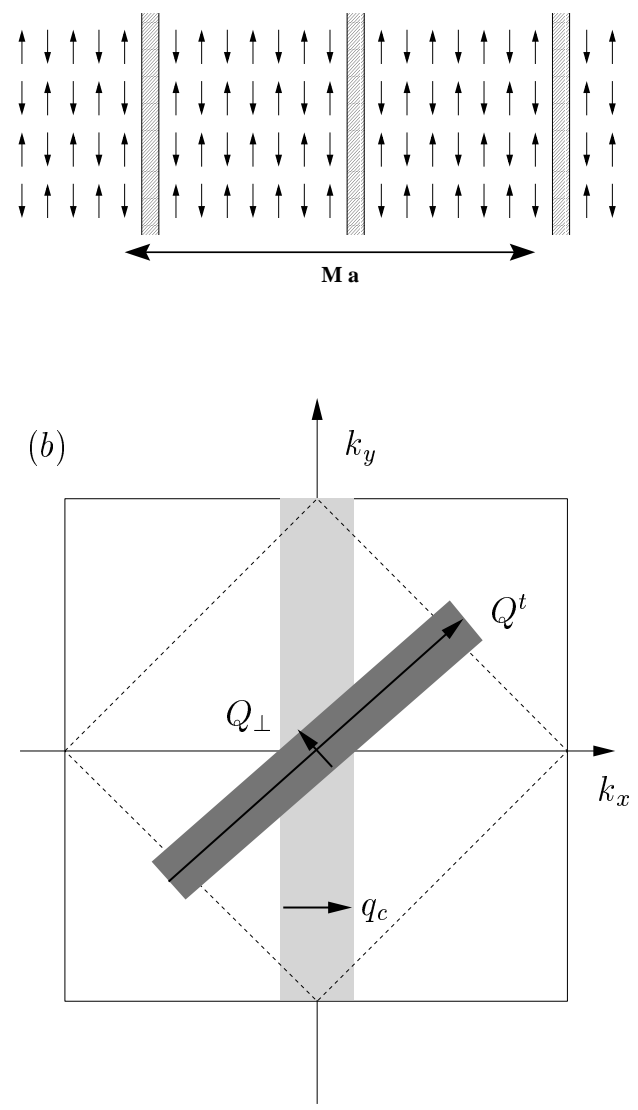

FIG.2 a) Sketch of a vertical stripe pattern where the shaded areas correspond to the charged domain walls. The commensurability $\mathrm{M}$ times the lattice constant a defines the periodicity of the structure. b) The large square represents the full Brillouin zone whereas the dark shaded rectangle depicts the reduced Brillouin zone for incommensurability $M=16$. The latter can be constructed from the reduced zone for charge order only (light shaded rectangle) which is rotated and divided in half due to antiferromagnetism. Since also the AF order is modulated the boundaries of the dark shaded rectangle are not parallel to those of the reduced zone for commensurate antiferromagnetism (dashed line).

and from the symmetry of $\mathrm{H}$ it turns out that the transformation matrices obey the relations $A_{n m}(k) \equiv$ $A_{n m}(k, \sigma)=(-1)^{n} A_{n m}(k,-\sigma)$ and $A_{n m}(k, \sigma)=$ $A_{M-n+2, M-m+2}^{*}(-k, \sigma)$.

We finally obtain the hamiltonian in diagonal representation as

$$
\begin{aligned}
H & =\sum_{k, \sigma} \sum_{n} E_{n}(k) f_{n}^{\dagger}(k, \sigma) f_{n}(k, \sigma) \\
& +\frac{2 N}{U} \sum_{p}\left(\left|\Delta_{p}\right|^{2}-\left|\chi_{2 p}\right|^{2}\right)
\end{aligned}
$$

where $E_{n}(k)$ denotes the dispersion of the n-th subband in the reduced BZ (dark shaded rectangle in Fig. $2 \mathrm{~b}$ ). The order parameters have to be determined selfconsistently.

In Fig. 3 we show a cut of the bandstructure through the reduced zone and the density of states for a stripe structure with commensurability $\mathrm{M}=16$ corresponding to the scattering vector $\mathbf{Q}^{\mathbf{t}}$ as defined above. As can be seen the spectrum is composed of 16 quasi onedimensional subbands. Since the choosen carrier concentration $\delta=0.13$ slightly exceeds the integer fraction $\delta=1 / 8$ the chemical potential is located within but near the top edge of the 7 th subband.

The following sections deal with the RPA fluctuations around the HF stripe state and it is quite instructive to consider the functional dependence of the ground state energy from the spin- and charge order parameters which is shown in Fig. 4. In fact, since RPA excitations can be derived from the expansion around the HF saddle-point in the density fluctuations (see e.g. 39 ) one can already qualitatively deduce from Fig. 4 the possible instabilities of the system. Note that for the $E\left(\Delta_{1}\right)$ curve the charge order parameter is adjusted selfconsistently while for the $E\left(\chi_{2}\right)$ curve the spin order parameter is taken at the saddle point. The $\left(\langle H\rangle, \Delta_{1}\right)$ curve displays the standard behavior of a second-order phase transition towards the SDW ground state with minimum at $\Delta_{1}=0.14 \mathrm{eV}$. On the other hand it turns out that the saddle-point of $\left(\langle H\rangle, \chi_{2}\right)$ corresponds to a maximum in the energy-order parameter space which is also obvious from the decoupling eq. (2) 40 . This simple picture already demonstrates that in the present framework charge order is completely due to the SDW instability.
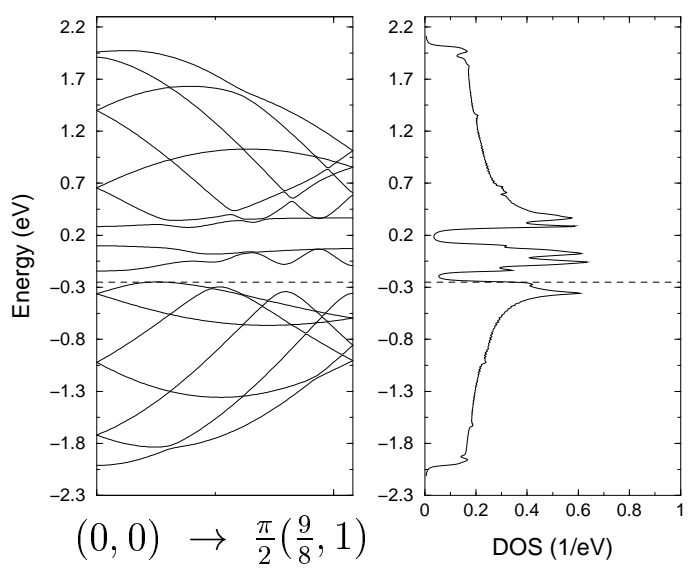

FIG.3 Band structure and density of states for a stripe array with commensurability $\mathrm{M}=16$ and $\mathrm{U} / \mathrm{t}=1.3$. The wave vector cut of the bandstructure is from $(0,0)$ along $\mathbf{Q}^{\mathbf{t}}$ (see Fig. $2 \mathrm{~b})$. The dashed line indicates the position of the chemical potential. 


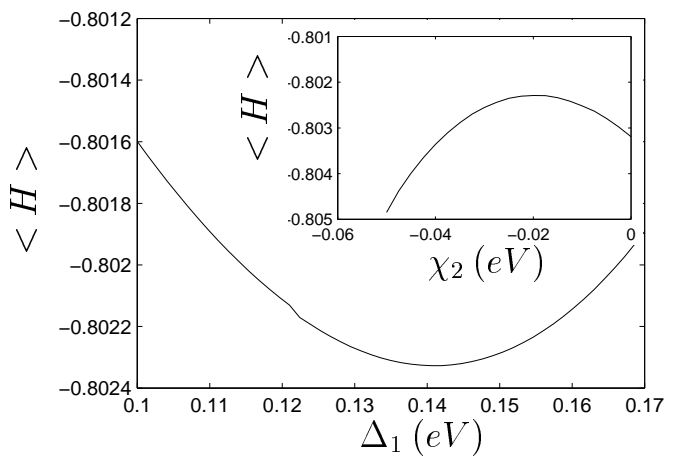

FIG.4 Free energy as a function of the SDW order parameter $\Delta_{1}$. The inset displays the free energy as a function of the CDW order parameter $\chi_{2}$.

Moreover, note that for $\chi_{2} \rightarrow 0$ the $\left(\langle H\rangle, \chi_{2}\right)$ curve has a finite derivative which signals the absence of critical fluctuations in the charge channel for the homogeneous system in contrast to models where the instability mechanism is due to frustrated phase separation 42.0 .

\section{B. Susceptibilities for general commensurability}

We start from the following definitions for charge and spin-density correlations functions

$$
\begin{aligned}
\chi_{m m^{\prime}}^{+-}\left(q, q^{\prime}, t\right) & =\frac{i}{N}\left\langle T S_{q+(m-1) Q^{t}}^{+}(t) S_{-q^{\prime}-\left(m^{\prime}-1\right) Q^{t}}^{-}(0)\right\rangle \\
\chi_{m m^{\prime}}^{\sigma \sigma^{\prime}}\left(q, q^{\prime}, t\right) & =\frac{i}{N}\left\langle T \rho_{q+(m-1) Q^{t}, \sigma}(t) \rho_{-q^{\prime}-\left(m^{\prime}-1\right) Q^{t}, \sigma^{\prime}}(0)\right\rangle, \\
\chi_{m m^{\prime}}^{00}\left(q, q^{\prime}, t\right) & =\sum_{\sigma \sigma^{\prime}} \chi_{m m^{\prime}}^{\sigma \sigma^{\prime}}\left(q, q^{\prime}, t\right), \\
\chi_{m m^{\prime}}^{z z}\left(q, q^{\prime}, t\right) & =\sum_{\sigma \sigma^{\prime}} \sigma \sigma^{\prime} \chi_{m m^{\prime}}^{\sigma \sigma^{\prime}}\left(q, q^{\prime}, t\right),
\end{aligned}
$$

where the wave vectors $q$ are within the reduced zone. Since the mean-field hamiltonian eq. (8) commutes with $\sum_{i} S_{i}^{z}$ the excitations in the longitudinal spin and charge channel are decoupled from the transverse channel where the solution of the RPA approximated Dyson equation for the latter case leads to

$$
\chi_{m m^{\prime}}^{+-}(q, \omega)=\left[1-U \chi_{m m^{\prime}}^{(0),+-}(q, \omega)\right]^{-1} \chi_{m m^{\prime \prime}}^{(0),+-}(q, \omega)
$$

The bare transverse stripe susceptibilities read as

$$
\begin{aligned}
& \chi_{m m^{\prime}}^{(0),+}(q, \omega)=\frac{1}{N} \sum_{k s t} \Gamma_{s t m}^{+}(\widetilde{k+q}, k) \Gamma_{t s m^{\prime}}^{-}(k, \widetilde{k+q}) \\
& {\left[\frac{n_{t}(\widetilde{k})\left[1-n_{s}(\widetilde{k+q})\right]}{\omega+E_{s}(\widetilde{k+q})-E_{t}(k)-i \eta}-\frac{n_{s}(\widetilde{k+q})\left[1-n_{t}(k)\right]}{\omega+E_{s}(\widetilde{k+q})-E_{t}(k)+i \eta}\right]}
\end{aligned}
$$

where we have introduced the vertex functions:

$$
\begin{aligned}
& \Gamma_{s t m}^{+}(\widetilde{k+q}, k)=\sum_{n} A_{\mathcal{N}, s}^{*}(\widetilde{k+q}) A_{n, t}(k)(-1)^{n-1} \\
& \Gamma_{t s m}^{-}(\widetilde{k, q+q})=\left(\Gamma_{s t m}^{+}(\widetilde{k+q}, k)\right)^{*}
\end{aligned}
$$

and $A_{n t}(k)$ is defined in Eq. (7). Here the index $\mathcal{N}=\mathcal{N}(n, m, k, q)$ defines the number of the BZ which contains the unreduced state $|k+q\rangle$ and $\widetilde{k+q}$ denotes the corresponding reduced wave vector.

We now turn to the evaluation of the correlation function in the longitudinal channel. In this case we start from the RPA decoupled Dyson equation for $\chi_{m m^{\prime}}^{\sigma \sigma^{\prime}}(q, \omega)$

$$
\chi_{m m^{\prime}}^{\sigma \sigma^{\prime}}(q, \omega)=\chi_{m m^{\prime}}^{(0), \sigma \sigma}(q, \omega)+U \chi_{m m^{\prime \prime}}^{(0), \sigma \sigma}(q, \omega) \chi_{m^{\prime \prime} m}^{-\sigma \sigma^{\prime}}(q, \omega)
$$

where

$$
\begin{aligned}
& \chi_{m m^{\prime}}^{(0), \sigma}(q, \omega)=\frac{1}{N} \sum_{k s t} \Gamma_{s t m}^{\sigma}(\widetilde{k+q}, k) \Gamma_{t s m^{\prime}}^{\sigma}(k, \widetilde{k+q}) \\
& {\left[\frac{n_{t}(k)\left[1-n_{s}(\widetilde{k+q})\right]}{\omega+E_{s}(\widetilde{k+q})-E_{t}(k)-i \eta}-\frac{n_{s}(\widetilde{k+q})\left[1-n_{t}(k)\right]}{\omega+E_{s}(\widetilde{k+q})-E_{t}(k)+i \eta}\right]}
\end{aligned}
$$

and the vertex functions are given by

$$
\begin{aligned}
& \Gamma_{\text {stm }}^{\sigma}(\widetilde{k+q}, k)=\sum_{n} A_{\mathcal{N}, s}^{*}(\widetilde{k+q}) A_{n, t}(k) \sigma^{n+\mathcal{N}} \\
& \Gamma_{t s m}^{\sigma}(\widetilde{k+q})=\left(\Gamma_{\text {stm }}^{\sigma}(\widetilde{k+q}, k)\right)^{*}
\end{aligned}
$$

Since we have to project $\chi_{m m^{\prime}}^{\sigma \sigma^{\prime}}(q, \omega)$ onto the charge and longitudinal spin sector we introduce the new matrices

$$
\kappa_{m m^{\prime}}=\frac{1}{2}\left(\begin{array}{ll}
\chi_{m m^{\prime}}^{\rho \rho} & \chi_{m m^{\prime}}^{\rho z} \\
\chi_{m m^{\prime}}^{z \rho} & \chi_{m m^{\prime}}^{z z}
\end{array}\right)=A^{-1} \frac{1}{2}\left(\begin{array}{cc}
\chi_{m m^{\prime}}^{\uparrow \uparrow} & \chi_{m m^{\prime}}^{\uparrow \downarrow} \\
\chi_{m m^{\prime}}^{\downarrow \uparrow} & \chi_{m m^{\prime}}^{\downarrow \downarrow}
\end{array}\right) A
$$

where

$$
A=\frac{1}{\sqrt{2}}\left(\begin{array}{rr}
1 & 1 \\
1 & -1
\end{array}\right)
$$

Within these definitions we can rewrite eq. (13) as a $2 M \times 2 M$ matrix equation for the charge and spin density correlations respectively

$$
\Xi=\left[1-U \Xi^{o} T_{z}\right]^{-1} \Xi^{o}
$$

and the matrices $T_{z}$ and $\Xi$ read as

$$
\Xi=\left(\begin{array}{cccc}
\kappa_{11} & \kappa_{12} & \cdots & \cdot \\
\kappa_{21} & \kappa_{22} & & \\
\cdot & & \cdot & \\
\cdot & & & \cdot \\
\cdot & & & .
\end{array}\right) ; T_{z}=\left(\begin{array}{ccccc}
\tau_{z} & 0 & 0 & \cdot \\
0 & \tau_{z} & 0 & \\
0 & 0 & \tau_{z} & \\
\cdot & & & \cdot \\
\cdot & & & & .
\end{array}\right) \text {. }
$$

Pbviously both charge and longitudinal spin excitations are strongly coupled except in the half-filled system where the ground state corresponds to a commensurate antiferromagnet. 


\section{Effective interactions for general commensurability}

Having determined the collective excitations in the striped phase we are now in the position to study the interactions of two holes within the same subband with opposite spin and momenta in the spin- and charge channel respectively. Note that the susceptibilities defined in eq. (9) acquire non-diagonal matrix elements in the stripe state, which implies the possibility of creating Cooper pairs with a finite momentum (which is a multiple of $\mathbf{Q}^{\mathbf{t}}$ ). However, for simplicity we will consider only scattering of zero momentum Cooper pairs and therefore neglect nondiagonal contributions of the susceptibilities $\left(m \neq m^{\prime}\right)$ to the interaction. Additionally we will cast these interactions in the form of effective hamiltonians thus restricting on the static limit with an appropriate frequency cutoff.

We find in the orientational spin-fluctuation channel

$$
\begin{aligned}
H^{+-} & =-\frac{U^{2}}{N} \sum_{\substack{k q \\
m s t}} \Gamma_{s t m}^{-}(\widetilde{k+q}, k) \Gamma_{s t m}^{+}(-\widetilde{k-}-,-k) \chi_{m m}^{+-}(q) \\
& \times f_{s}^{+}(\widetilde{k+q},-\sigma) f_{s}^{+}(\widetilde{k-q}, \sigma) f_{t}(k, \sigma) f_{t}(-k,-\sigma)
\end{aligned}
$$

in the charge-fluctuation channel

$$
\begin{aligned}
H^{\rho \rho} & =-\frac{U^{2}}{N} \sum_{\substack{k q \\
m s t}} \Gamma_{s t m}^{\sigma}(\widetilde{k+q}, k) \Gamma_{s t m}^{-\sigma}(-\widetilde{k-}-q,-k) \chi_{m m}^{\rho \rho}(q) \\
& \times f_{s}^{+}(\widetilde{k+q},-\sigma) f_{s}^{+}(-\widetilde{k-} q, \sigma) f_{t}(k, \sigma) f_{t}(-k,-\sigma) \quad(21)
\end{aligned}
$$

and in the amplitude spin-fluctuation channel

$$
\begin{aligned}
H^{z z} & =-\frac{U^{2}}{N} \sum_{\substack{k q \\
m m s t}} \Gamma_{s t m}^{\sigma}(\widetilde{k+q}, k) \Gamma_{s t m}^{-\sigma}(-\widetilde{k-q} q,-k) \chi_{m m}^{z z}(q) \\
& \times f_{s}^{+}(\widetilde{k+q},-\sigma) f_{s}^{+}(\widetilde{k-q}, \sigma) f_{t}(k, \sigma) f_{t}(-k,-\sigma) .
\end{aligned}
$$

In order to illustrate the behavior of the vertex functions in the various channels let us consider the case of well developed stripe order (i.e. large charge- and spin order parameters). In this limit we can as a first approximation neglect the kinetic energy in eq. (6) and the transformation eq. (7) takes the form

$$
A_{n t}(k) \approx \frac{1}{\sqrt{M}} \exp \left[i \frac{2 \pi}{M} n t\right] .
$$

As a result the vertex contribution in the transverse spin channel is given by

$$
\Gamma_{s t m}^{-}(k, q) \Gamma_{s t m}^{+}(k, q)=(-1)^{(m-1)} \delta_{s, t+\frac{M}{2}}
$$

and thus the coupling to spin-flip scattering vanishes for intraband transitions.

On the other hand the intraband coupling in the charge and longitudinal spin channel survives 41 and can be expressed in the form

$$
\Gamma_{s t m}^{\uparrow}(k, q) \Gamma_{s t m}^{\downarrow}(k, q)=(-1)^{(m-1)} \delta_{s, t}
$$

Now remember that the index $m$ in $\chi_{m m}(\mathbf{q})$ labels the multiple of $\mathbf{Q}^{\mathbf{t}}$ which has to be added to the reduced transfered momentum $\tilde{\mathbf{q}}$, i.e. the transfered momentum in the full $\mathrm{BZ}$ is $\mathbf{q}=\tilde{\mathbf{q}}+(m-1) \mathbf{Q}^{\mathbf{t}}$. Thus an odd (even) index $m$ corresponds to enhanced charge (spin) susceptibilities which therefore in general mediate attractive (repulsive) interactions respectively. In addition upon assuming that the charge (spin) susceptibility has some finite contribution for odd (even) $m$ only, one recovers the result of Ref 35 that the effective interaction in these channels becomes proportional to the commensurability of the charge modulation, i.e. $\sum_{m} \Gamma_{s t m}^{\uparrow}(k, q) \Gamma_{s t m}^{\downarrow}(k, q)= \pm M / 2$.

\section{RESULTS}

We now apply the formalism developed above to the calculation of the effective interaction within the $\mathrm{HF}$ stripe state of the two-dimensional Hubbard model. We restrict ourselves to the domain wall considered in Sec. IIa, i.e. commensurability $M=16$ for which the total scattering vector is $Q^{t}=\left(\pi, \pi+\frac{\pi}{8}\right)$. Fig. 5 displays the different RPA susceptibilities for $\omega=0$ and $\omega=25 \mathrm{meV}$ respectively. Note that the q-scans for the magnetic susceptibilities are along the $\left(q_{x}, q_{y}=\pi\right)$ axis whereas the charge susceptibility is shown along $\left(q_{x}, q_{y}=0\right)$. The structure in $\chi^{+-}$is dominated by the Goldstone pole at $Q^{t}$ due to the breaking of spin-rotational invariance in the stripe state. As can be seen from Fig.6 the mode splits with increasing frequency and the two branches result from the defolding of the reduced BZ (Ref.28). Moreover beyond the gap energy (roughly corresponding to $\Delta_{1}$ ) the magnetic excitations rapidly lose intensity.

The longitudinal spin and charge channel do not display a physical pole at $\omega=0$. However, a mode like feature is present at $Q^{t}$ for the amplitude fluctuations and at $2 Q^{t}$ for the charge susceptibility which reflects the enhanced susceptibility of the stripe state to perturbations with the respective wave numbers. Since both charge and spin are coupled within the RPA formalism the respective excitations follow the same dispersion in $(q, \omega)$-space (Fig. 6) but since the charge 'mode' appears as a second harmonic to the longitudinal spin excitation its intensity is significantly lower. It is interesting to observe that at the energy $\left(\approx \Delta_{1}\right)$ where the longitudinal spin fluctuations become commensurate the charge fluctuations show the periodicity of the static spin modulation (i.e. $\mathbf{q}_{\mathbf{s}}$ ) which again is a consequence of the defolding of the reduced BZ. Note that this energy does not correspond to the maximum in the intensity, which appears for slightly lower frequencies. 

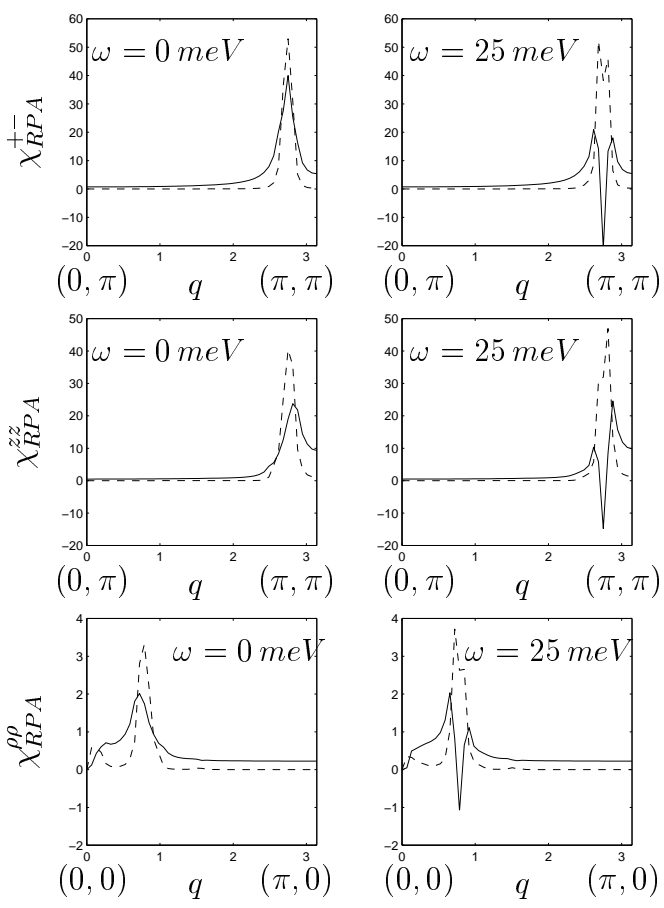

FIG.5 The momentum dependence of the RPA susceptibilities $\chi_{R P A}^{+-}, \chi_{R P A}^{z z}, \chi_{R P A}^{\rho \rho}$ in the stripe state for two frequences $\omega=0$ (left column) and $\omega=25 \mathrm{meV}$ (right column); Solid: real part, Dashed: imaginary part; first and second lines represent the scans along path $(0, \pi)-(\pi, \pi)$, third line represents the scan along path $(0,0)-(\pi, 0)$.

Finally, in order to determine the efficieny of the pairing in the various channels we have to calculate the vertex functions which determine the coupling of the charge carriers to the corresponding correlation functions. Generally speaking the vertex functions determine the overall sign of the effective interaction in the Brillouin zone. Let us briefly illustrate this effect in the limit of a commensurate spin-density wave $(M=2)$ where the vertex functions can be calculated analytically. It is then shown below that the qualitative behavior of the coupling still holds for higher commensurability. Note that the pairing interaction mediated by amplitude fluctuations of AF order constitutes the basis of the so called "spin bag mechanism to high- $\mathrm{T}_{c}$ supgrconductivity" as proposed by Schrieffer et al. in Ref.22. In this case the antiferromagnetic scattering $Q=(\pi, \pi)$ doubles the unit cell and we denote conduction and valence band by the superscripts $c$ and $v$ respectively. The corresponding energies are given by $E_{k}^{c / v}= \pm E_{k}= \pm \sqrt{\Delta^{2}+\epsilon_{k}^{2}}$ and $\Delta$ is the SDW order parameter.

Restricting ourselves on the 'hole doped' case the relevant coupling functions can be written within our notation as

$$
\begin{aligned}
\Gamma_{v v m}^{\sigma} \Gamma_{v v m}^{-\sigma} & =\frac{(-1)^{m-1}}{2}\left[1-\frac{(-1)^{m} \epsilon_{k} \epsilon_{k+q}-\Delta^{2}}{E_{k} E_{k+q}}\right] \\
\Gamma_{v v m}^{+} \Gamma_{v v m}^{-} & =\frac{(-1)^{m-1}}{2}\left[1-\frac{(-1)^{m} \epsilon_{k} \epsilon_{k+q}+\Delta^{2}}{E_{k} E_{k+q}}\right]
\end{aligned}
$$

Obviously there is a difference in sign between small q- $(m=1)$ and large q- $(m=2)$ scattering. Since in an antiferromagnet the magnetic susceptibilities are peaked at $(\pi, \pi)$ the attractive interaction for small momentum transfer (as anticipated in the spin-bag mechanism) is accompanied by a large repulsion at $\mathbf{q} \sim \mathbf{Q}$ (see e.g. Ref.32,36 for a more detailed analysis of this point).

Let us now consider the case of commensurability $\mathrm{M}=16$. As already mentioned the considered hole doping $\delta=0.13$ is slightly above the 'magic' concentration $1 / 8$ so that the Fermi level is located within the 7 th subband in the reduced BZ.

In Fig. 7 we have plotted the corresponding vertex functions for intraband scattering which for convenience are defolded along $\left(q_{x}, 0\right)$ and $\left(q_{x}, \pi\right)$. It turns out that also in this case the coupling around odd multiples of $Q^{t}$ is negativ whereas it is positive around the even harmonics. Thus also in the stripe phase the large q scattering which is dominated by the magnetic fluctuations is repulsive whereas attraction predominantly arises from the coupling to charge fluctuations. Naturally for stripes the latter are much more pronounced in comparison to the spin-bag model. With regard to the effective hamiltonian eqs. (20 22) the cutoff of the pairing potential is an important parameter in determining the interaction strength. From the right panel of Fig. 5 we conclude that for the relevant wave-vectors near the 'modes' the cutoff energy approximately is of the order of the charge order parameter $\chi_{2}$.

Besides the enhancement of charge fluctuation mediated pairing in the stripe phase the contribution of spinflip scattering to the interaction is also significantly different from the spin-bag approach. First note that the coupling to the transverse susceptibility for large momenta (upper right panel of Fig. (7)) is by two orders of magnitude smaller than the corresponding coupling to charge and longitudinal spin fluctuations. In addition it vanishes at the momentum of the Goldstone pole i.e. $q=Q^{t}$. For the commensurate $M=2$ case this can be seen directly from eq. 27) in the limit $\mathbf{q} \rightarrow(\pi, \pi)$ (note that in our reducednotation this is equivalent to $\mathbf{q}=0$ in eq. (27). In Ref 22 this fact led to some confusion concerning the relevance of the transverse fluctuations within thespinbag approach. However, it has been shown in Ref.23 that the vanishing of the vertex is cancelled by the gapless Goldstone pole of $\chi^{+-}(Q)$ and as a result the interaction in the spin-flip channel around $(\pi, \pi)$ becomes comparable to the pairing from the amplitude fluctuations in the spin-bag scenario. 

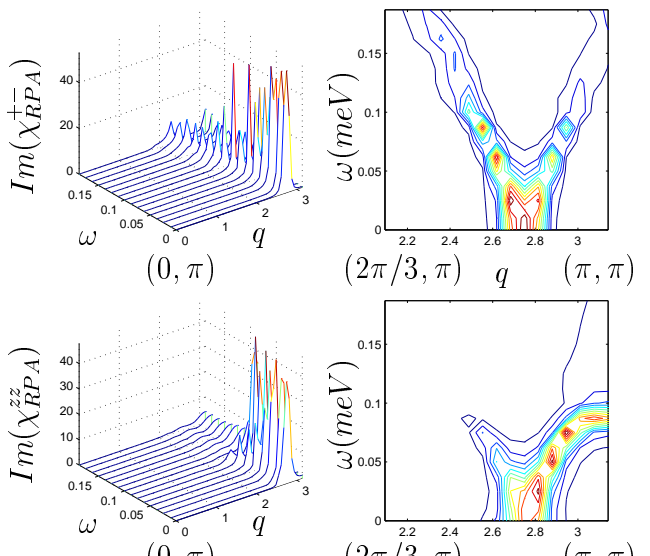

$(0, \pi)$
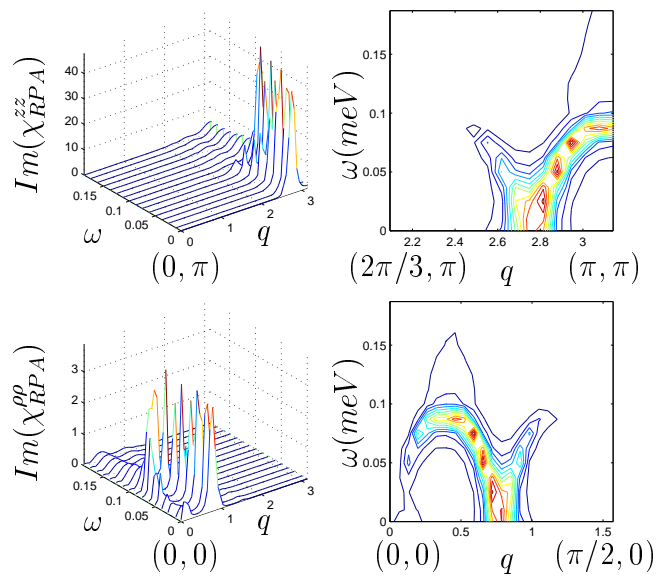

FIG.6 The momentum and frequency dependences of the imaginary parts of the RPA susceptibilities $\chi_{R P A}^{+-}, \chi_{R P A}^{z z}, \chi_{R P A}^{\rho \rho}$ in the stripe state; right column shows the intensity contour along $(0, \pi)-(\pi, \pi)$ for $\operatorname{Im}\left(\chi_{R P A}^{+-}\right), \operatorname{Im}\left(\chi_{R P A}^{z z}\right)$ and along $(0,0)-(\pi, 0)$ for $\operatorname{Im}\left(\chi_{R P A}^{\rho \rho}\right)$..

In order to study the coupling to transverse fluctuations for commensurability $M=16$ we have performed a numerical expansion of the vertex and the transverse susceptibility around $q=Q^{t}$. Contrary to the low commensurability case one finds that the contribution from the transverse channel to the pairing is only $\approx 5 \%$ of that of the spin amplitude fluctuations. We consider this as a consequence of the spatial separation of charge carriers and spin fluctuations in the stripe model.

\section{CONCLUSION}

To summarize, we have calculated the effective interactions arising from the RPA fluctuations around the HF stripe phase of the two-dimensional Hubbard model. Since our approach is a generalization of Ref.22 to higher commensurabilities the general features resemble closely those of the spin-bag mechanism. However, significant differences arise due to the inhomogeneous charge distribution in the stripe state where the holes are confined to the domain walls separating antiphase AF regions. As a result the charge correlations are enhanced for wave-vectors corresponding to the stripe periodicity resulting in a much more pronounced small q attractive pairing as compared to the spin-bag approach where the system is translationally invariant with respect to the charge degrees of freedom. Due to the fact that HF theory generates the charge order as a second harmonic of the longitudinal spin modulation the pairing is still dominated by the large $q$ repulsion due to magnetic fluctuations. However, in models where the stripe instability is driven by the charge an analogous approach should yield a comparable interaction strength in spinand charge channels respectively. Thus depending on the repulsive contribution due to magnetic scattering either a d-wave or anisotropic s-wave SC order parameter minimizes the free energy of the system. Obviously non of these order parameters will be really realized in a purely one-dimensional stripe phase as considered in the present paper, however, our investigations can be considered as complementary to Ref. 43 where the coexistence between incommensurate antiferromagnetism and anisotropic superconductivity has been investigated. In this context the effective interaction derived above may provide a microscopic origip for the superconducting correlations considered in Ref.43. Anyhow, in order to have true d-wave order one has to anticipate a scenario where the stripes fluctuate between the $\mathrm{x}$ - and $\mathrm{y}$ - directions in order to restore the two-dimensional symmetry. In principle one could symmetrize the pairing interaction derived above and use it in a BCS-type approach in order to evaluate the superconducting gap. Alternatively, one could consider the interactions arising from the fluctuatipns of an eggbox-type domain wall structure as in Ref 44 in order to mimic orientational stripe fluctuations. Furthermore it has been proposed 45 that domains of stripes running along the crystalographic directions can couple via the twin boundaries which also leads to 'symmetric' d-wave superconductivity.
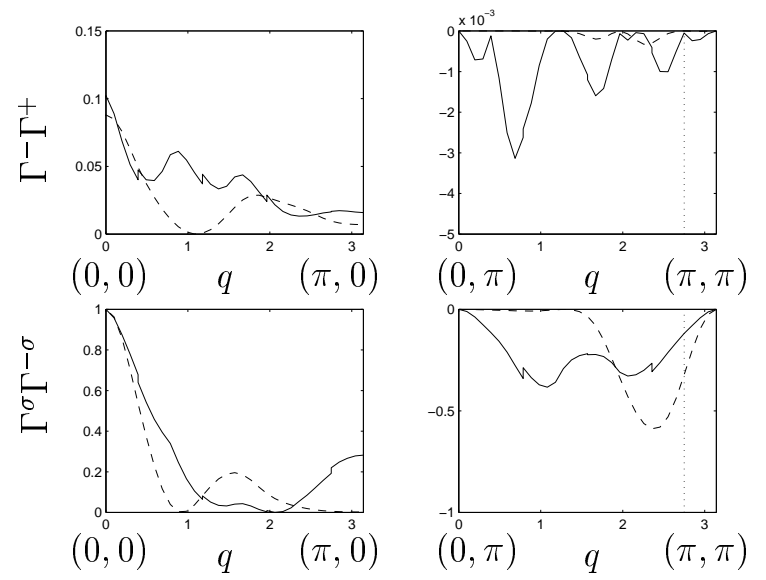

FIG.7 The vertex functions $\Gamma_{77 m}^{-}(k, q) \Gamma_{77 m}^{+}(k, q)$ and $\Gamma_{77 m}^{\sigma}(k, q) \Gamma_{77 m}^{-\sigma}(k, q)$ for two points in the reduced BZ: $k=$ $(-\pi / 1.6,-\pi / 2.3)$, solid curve; $k=(0,0)$, dashed curve; The dotted line indicates the position of $\mathbf{Q}^{\mathbf{t}}$. 
However, within the obvious limitations of our approach which we have already discussed in the introduction a quantitative evaluation of the superconducting gap would not be very instructive and beyond the scope of the present paper. Here our purpose is to simply outline the general features of stripe fluctuation induced pairing and to eventually supplement considerations within more sophisticated approaches.

${ }^{1}$ P. C. Hammel, A. P. Reyes, Z. Fisk, M. Takigawa, J. D. Thompson, K. H. Heffner, and S. W. Chong, Phys. Rev. B 42, 6781 (1990).

${ }^{2}$ J. Mesot, P. Allenspach, U. Staub, A. Furrer, and H. Mutka, Phys. Rev. Lett. 70, 865 (1993).

${ }^{3}$ G. B. Teitel'baum, B. Büchner, H.de Gronckel, Phys. Rev. Lett. 84, 2949 (2000).

${ }^{4}$ A. Suter, M. Mali, J. Roos, D. Brinkmann, J. Karpinski, and E. Kaldis, Phys. Rev. B 56, 5542 (1997); I. Eremin, M. Eremin, S. Varlamov, D. Brinkmann, M. Mali, and J. Roos, Phys. Rev. B 56, 11305 (1997).

${ }^{5}$ S. Krämer, M. Mehring, Phys. Rev. Lett. 83, 396 (1999).

${ }^{6}$ A. W. Hunt, P. M. Singer, K. R. Thurber, and T. Imai, Phys. Rev. Lett. 82, 4300 (1999).

7 J.Zaanen and O.Gunnarsson, Phys. Rev. B40, 7391 (1989).

${ }^{8}$ D.Poilblanc and T.M.Rice, Phys. Rev. B39, 9739 (1989).

${ }^{9}$ J. M. Tranquada, B. J. Sternlieb, J. D. Axe, Y. Nakamura, and S. Uchida, Nature 375, 561 (1995). Y. Nakamura, and S. Uchida, Nature 375, 561 (1995).

${ }^{10}$ B. Normand, A. P. Kampf, Phys. Rev. B 64, 024521 (2001).

${ }^{11}$ K. Yamada, C. H. Lee, K. Kurahashi, J. Wada, S. Wakimoto, S. Ueki, H. Kimura, Y. Endoh, S. Hosoya, G. Shirane, R. J. Birgeneau, M. Greven, M. A. Kastner, and Y. J.Kim, Phys. Rev. B 57, 6165 (1998).

${ }^{12}$ M. Arai, T. Nishijima, Y. Endoh, T. Egami, S. Tajima, K. Tomimoto, Y. Shiohara, M. Takahashi, A. Garrett, S. Bennington, Phys. Rev. Lett. 83, 608 (1999).

${ }^{13}$ H. A. Mook and F. Doĝan, Nature 401, 145 (1999).

${ }^{14}$ E.S. Bozin, G. H. Kwei, H. Takagi, and S. J. L. Billinge, Phys. Rev. Lett. 84, 5856 (2000).

${ }^{15}$ M. Arai, Proceedings 3rd International Conference on 'Stripes and high $T_{c}$ superconductivity', Roma (2000), will be published in Int. J. Mod. Phys. (2000).

${ }^{16}$ C. Castellani, C. Di Castro, and M. Grilli, Phys. Rev. Lett. 75, 4650 (1995); C. Castellani, C. Di Castro, and M. Grilli, Z. Phys. B 103, 137 (1997).

17 J. L. Tallon, J. W. Loram, G. V. M. Williams, J. R. Cooper, I. R. Fisher, J. D. Johnson, M. P. Staines, C. Bernhard, Phys. Stat. Sol. B 215, 531 (1999); J. L. Tallon, G. V. M. Williams, and J. W. Loram, Physica C 338, 9 (2000).
${ }^{18}$ G. S. Boebinger, Yoichi Ando, A. Passner,T. Kimura, M. Okuya, J. Shimoyama, K. Kishio, K. Tamasaku, N. Ichikawa, S. Uchida, Phys. Rev. Lett. 77, 5417 (1996).

19 A. Perali, C. Castellani, C. Di Castro, and M. Grilli, Phys. Rev. B 54, 16216 (1996).

${ }^{20}$ C. A. Balseiro and L. M. Falicov, Phys. Rev. B 20, 4457 (1979).

21 M.V.Eremin, I.A.Larionov, and S.V.Varlamov. Physica B 259-261, 456 (1999).

22 J.R.Schrieffer, X.G.Wen, and S.C.Zhang, Phys. Rev. B39, 11663 (1989).

23 D.M.Frenkel and W.Hanke, Phys. Rev. B42, 6711 (1990).

${ }^{24}$ J. Zaanen and M. Oleś, Ann. Physik 5, 224 (1996).

${ }^{25}$ G.Seibold, C.Castellani, C.Di Castro, M.Grilli, Phys.Rev. B58, 13506 (1998).

${ }^{26}$ A. I. Lichtenstein, M. Fleck, A. M. Oleś, and L. Hedin, in Stripes and Related Phenomena, edited by A. Bianconi and N. L. Saini, Kluwer Academic (2000).

${ }^{27}$ H.J.Schulz, Phys. Rev. L64, 1445 (1990);H.J.Schulz, J.Phys.France 50, 2833 (1989).

${ }^{28}$ E.Kaneshita, M.Ichioka and K.Machida, unpublished, cond-mat/0005466.

29 O.Zachar, unpublished, cond-mat/0001217.

${ }^{30}$ for a recent analysis of this scenario including fluctuation effects see e.g. E. Arrigoni and G.C.Strinati,Eur. Phys. J. B19, (2001) and references therein.

31 A.W.Overhauser, Phys. Rev. 128, 1437 (1962).

32 A.V.Chubukov and D.M.Frenkel , Phys. Rev. B46, 11884 (1992).

33 M.Inui and P.B.Littlewood, Phys. Rev. B 44, 4415 (1991).

${ }^{34}$ O.Zachar, S.A.Kivelson and V.J.Emery, Phys. Rev. B 57, 1422 (1998).

${ }^{35}$ G.Seibold and S.Varlamov, Phys. Rev. B60, 13056 (1999).

36 A.P.Kampf, Physics Reports 249, 219 (1994).

${ }^{37} \mathrm{M}$.

Matsuda, M.Fujita, K.Yamada, R.J.Birgeneau, M.A.Kastner, H.Hiraka, Y.Endoh, S.Wakimoto, G.Shirane, Phys. Rev. 62, 9148 (2000).

38 J. Tranquada, Physica B 241-243, 745 (1997).

39 J.-P. Blaizot and G. Ripka, Quantum Theory of Finite Systems, (The MIT Press, 1986).

${ }^{40}$ Since the maximum is the only extremum of the $\left(\langle H\rangle, \chi_{2}\right)$ curve the system cannot acquire a self-consistent state with lower energy. Note that in this case the free energy is not a Landau functional which would have minima at finite order parameter values.

${ }^{41}$ Note that within our analysis the chemical potential is located within a subband so that intraband excitations are still possible.

${ }^{42}$ V.J.Emery and S.A.Kivelson, Physica C 209, 597 (1993).

${ }^{43}$ I. Martin, G. Ortiz, A. V. Balatsky, and A. R. Bishop, cond-mat/0009067.

44 G.Seibold, F.Becca, F.Bucci, C.Castellani, C.Di Castro, and M.Grilli, Eur.Phys.J. B 513, 87 (2000).

${ }^{45}$ A. H. Castro Neto, cond-mat/0102281. 